\title{
Engineering reactors for catalytic reactions
}

\author{
VIVEK V RANADE* \\ Chemical Engineering and Process Development Division, CSIR - National Chemical Laboratory, \\ Pune 411 008, India \\ e-mail: vv.ranade@ncl.res.in
}

MS received 2 July 2013; revised 26 December 2013; accepted 30 December 2013

\begin{abstract}
Catalytic reactions are ubiquitous in chemical and allied industries. A homogeneous or heterogeneous catalyst which provides an alternative route of reaction with lower activation energy and better control on selectivity can make substantial impact on process viability and economics. Extensive studies have been conducted to establish sound basis for design and engineering of reactors for practising such catalytic reactions and for realizing improvements in reactor performance. In this article, application of recent (and not so recent) developments in engineering reactors for catalytic reactions is discussed. Some examples where performance enhancement was realized by catalyst design, appropriate choice of reactor, better injection and dispersion strategies and recent advances in process intensification/multifunctional reactors are discussed to illustrate the approach.
\end{abstract}

Keywords. Catalyst; reactors; effectiveness; hydrodynamics; engineering.

\section{Introduction}

Chemicals and allied industries manufacture products which are essential for realizing and sustaining modern societies. Chemical (and biological) transformations necessary to make these essential products often involve use of catalysts. A catalyst (can be homogeneous or heterogeneous) reduces activation energy barrier to transformations and facilitates better control on selectivity. Development and selection of the right catalyst therefore can make substantial impact on process viability and economics. Besides the right catalyst, it is essential to develop the right reactor type and process intensification strategies for effective translation of laboratory process to practise. Reaction and reactor engineering which deal with these aspects therefore play a crucial role in chemical and allied process industries. In this article, application of recent (and not so recent) developments in engineering reactors for catalytic reactions is discussed.

Engineering of reactors includes all the activities necessary to evolve best possible hardware and operating protocol of reactor to carry out the desired transformation of raw materials (or reactants) into valueadded products. Any catalytic reactor has to carry out

*For correspondence several functions such as bringing reactants into intimate contact with active sites on catalyst (to allow chemical reactions to occur), providing an appropriate environment (temperature and concentration fields) for adequate time and allowing for removal of products from catalyst surface. A reactor engineer has to ensure that the evolved reactor hardware and operating protocol satisfy various process demands without compromising safety, environment and economics. Naturally, successful reactor engineering requires bringing together better chemistry (thermodynamics, catalysis (replace reagent-based processes), improved solvents (supercritical media, ionic liquids), improved atom efficiency, prevent wasters - leave no waste to treat) and better engineering (fluid dynamics, mixing and heat and mass transfer, new ways of process intensification, computational models and real-time process monitoring and control).

Several tools for modelling of chemical kinetics and reactions are already well-developed and routinely used in practice for facilitating engineering of reactors. Several excellent textbooks discussing these classical reaction engineering tools and practices are available. ${ }^{1-4}$ In this article, we have used these classical texts as a starting point and discuss some of the recent advances in reaction/reactor engineering practices for optimizing catalytic reactors. The scope is restricted to discussion of catalytic reactors. The vast field of catalysts and catalysis is briefly discussed in the following section. 
Key issues in and approach for engineering of catalytic reactors are then discussed. Some examples where performance enhancement was realized by catalyst design, appropriate choice of reactor, better injection and dispersion strategies and recent advances in process intensification/multifunctional reactors which were used for performance enhancement are discussed to illustrate the approach.

\section{Catalyst and catalytic reactions}

It will be prudent to briefly summarize and recapitulate key aspects of catalyst and catalytic reactions before other engineering aspects are discussed. A catalyst is a substance which provides an alternative route of reaction where the activation energy is lower without actually taking part in the reaction (see figure 1). Catalysts do not affect chemical equilibrium associated with a reaction. They merely change the rate of reactions. Catalysts are classified in a variety of ways. The commonly used classification by reaction engineers is based on number of phases as:

- Homogenous catalysis (catalyst and substrate in same phase)

- Heterogeneous catalysis (solid catalysis and substrate is a gas and/or liquid).

Homogeneous catalyst typically forms a complex with one of the reactants which eventually transforms it into the product after interacting with other reactants. The process is essentially similar to homogeneous reactions in absence of catalyst and is often controlled by mixing of reactants and catalyst species at molecular level.
In contrast to this, in heterogeneous catalyst, several additional steps are involved along with reaction occurring on catalyst surface such as (see figure 2):

- External diffusion towards catalyst pellet

- Internal diffusion towards catalyst surface

- Molecular adsorption on catalyst surface

- Surface reaction

- Desorption from catalyst surface

- Internal diffusion away from catalyst surface

- External diffusion away from catalyst pellet.
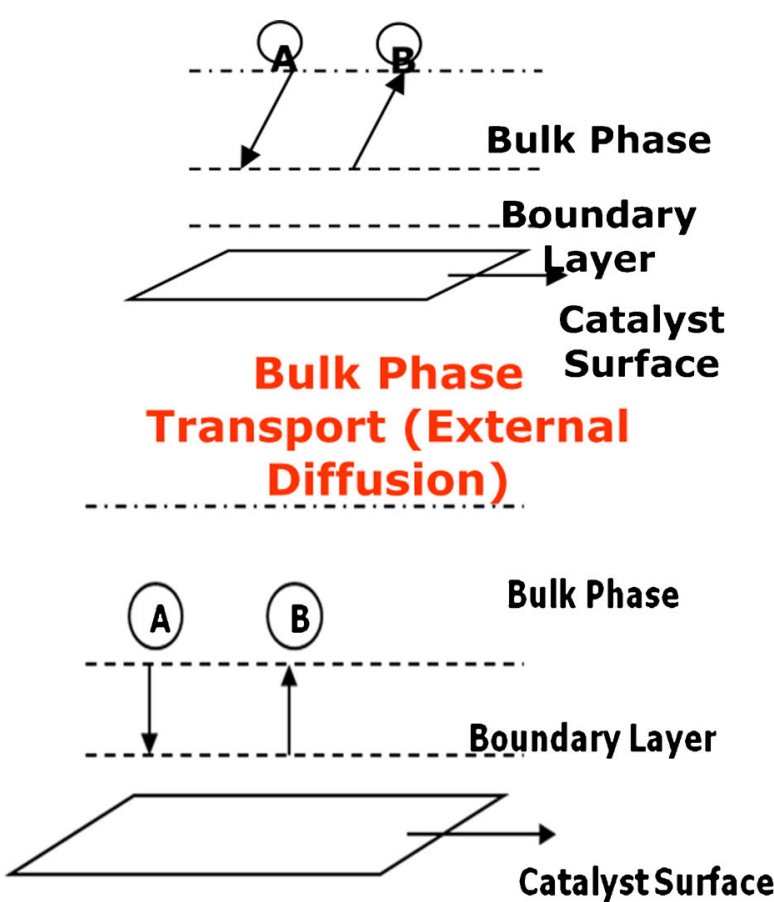

Film Transport (Internal Diffusion)

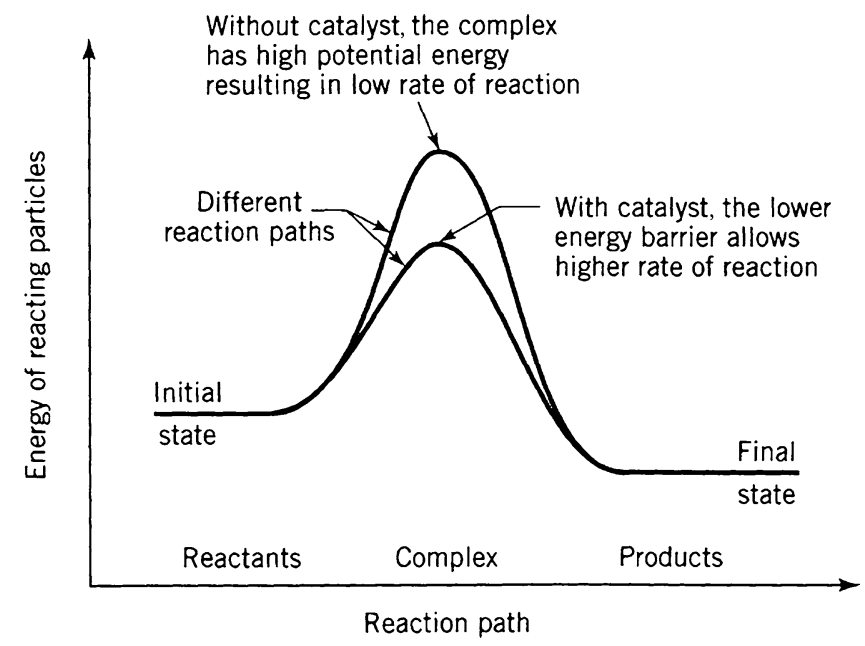

Figure 1. Role of catalyst. ${ }^{4}$

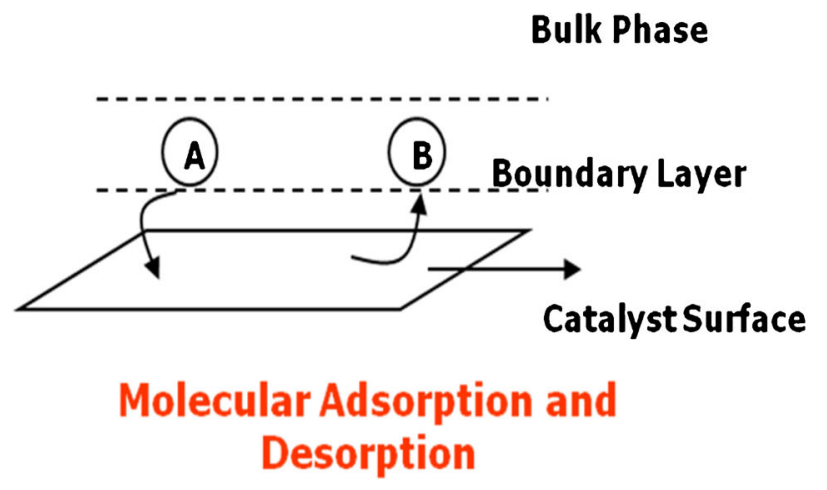

Figure 2. Steps in heterogeneous catalysis. 
These steps need to be understood in order to select appropriate reactor and operating strategy. This will be discussed later in this article.

Another important aspect of the catalyst is possible catalyst deactivation. The most typical causes of deactivation of heterogeneous catalyst are:

- Ageing: deactivation resulting from changes in structure

- Sintering: increase in average size of the crystallites due to coalescence of small solids on continued usage of catalyst

- Coking: deposition of high molecular weight Carbon-Hydrogen compounds on catalyst surface

- Poisoning: inhibitory substances bind to the active sites on catalytic surface.

Catalytically active complex in homogeneous catalysis may similarly get deactivated due to structural changes in the active complex as well as poisoning because of binding with inhibitory substances. A reactor engineer has to account for activation as well as deactivation of catalyst while designing suitable reactor for carrying out catalytic reactions. Basic aspects of practical reactor engineering are briefly discussed here.

\section{Practical reactor engineering}

Reactor engineering involves establishing a relationship between reactor hardware and operating protocols with various performance issues as listed in table 1 .

A general reactor engineering methodology is shown in figure 3. Based on the available information about the chemistry and catalysis of the process under consideration, the first step of reactor engineering is to select a suitable reactor type. In catalytic reactors, multiple phases are almost always involved (see examples cited in earlier studies. ${ }^{5-9}$ There are several types of reactors used for such catalytic and multiphase applications. Broadly, these reactors may be classified based on presence of phases as follows.

- Gas-liquid reactors: Stirred reactors, bubble column reactors, packed columns, loop reactors

- Gas-liquid-solids reactors: Stirred slurry reactors, three-phase fluidized bed reactors (bubble column slurry reactors), packed bubble column reactors, trickle bed reactors, loop reactors

- Gas-solid reactors: Fluidized bed reactors, fixed bed reactors, moving bed reactors

Existence of multiple phases opens up a variety of choices in bringing these phases together to react. Krishna and $\mathrm{Sie}^{10}$ have discussed a three-level approach for reactor design and selection (see figure 4).

- Strategy level I: Catalyst design strategy

- gas-solid systems: catalyst particle size, shape, porous structure, distribution of active material

○ gas-liquid systems: choice of gas-dispersed or liquid-dispersed systems, ratio between liquid-phase bulk volume and liquid-phase diffusion layer volume

- Strategy level II: Injection and dispersion strategies

o reactant and energy injection: batch, continuous, pulsed, staged. . .

o state of mixedness of concentrations and temperature

- separation of product or energy in situ

○ contacting flow pattern: co-, counter-, crosscurrent

- Strategy level III: Choice of hydrodynamic flow regime

o packed bed, bubbly flow, churn-turbulent regime, dense-phase or dilute-phase riser transport

Table 1. Reactor engineering.

\begin{tabular}{|l|}
\hline Reactor performance \\
\hline Conversion and selectivity \\
\hline Product quality \\
\hline Catalyst activity and life \\
\hline Stability and operability \\
\hline Safety \\
\hline Environmental impact \\
\hline
\end{tabular}

\begin{tabular}{l} 
Hardware and operating protocol \\
Reactor configuration: Size \& shape, feed \\
\& exit nozzles \\
\hline $\begin{array}{l}\text { Mode of operation: Batch, semi-batch, } \\
\text { continuous }\end{array}$ \\
\hline Start-up \& shut-down protocols \\
\hline Operating conditions: Flow rate, pressure, \\
temperature, flow regime, RTD \\
\hline $\begin{array}{l}\text { Reactor Internals: Baffles, heat transfer } \\
\text { coils, distributors }\end{array}$ \\
\hline
\end{tabular}




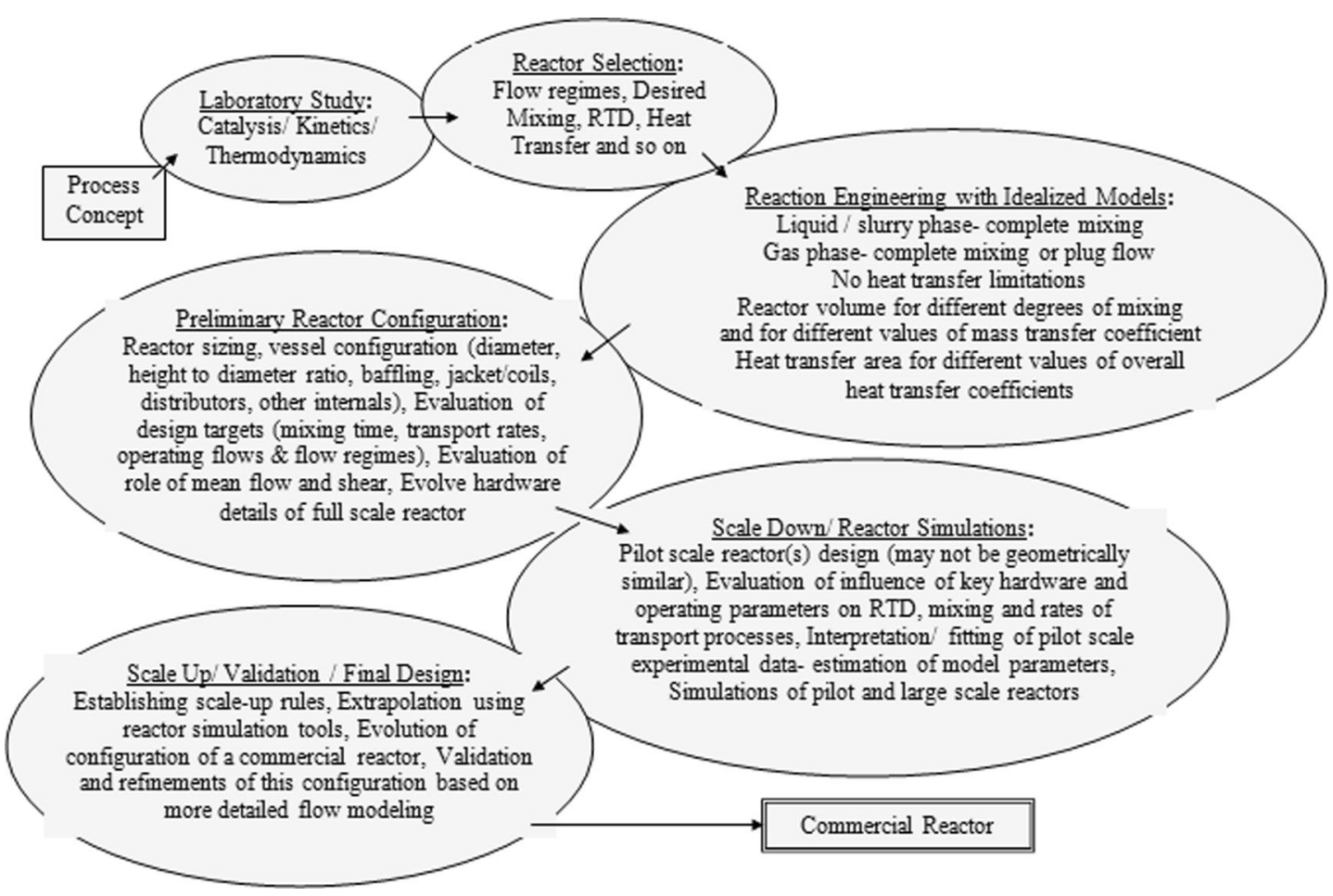

Figure 3. Reactor engineering methodology. ${ }^{17}$

Besides these considerations for selecting appropriate reactor and mode of operation, there are several other factors which need to be considered while designing a catalytic reactor. Some of the key issues are:

- Understanding gas-liquid and liquid-solid transport processes: Mass and heat transfer across multiple phases play a crucial role in determining performance of multiphase catalytic reactors. The key issues are shown schematically in figure 5 . Ramachandran and Chaudhari have elucidated these points very well in their classic book on three-phase catalytic reactors and interested readers should consult the original book. ${ }^{5}$

- Understanding intra-particle transport processes: mass and heat transfer effects are important even on a catalyst particle scale. Most of the catalysts

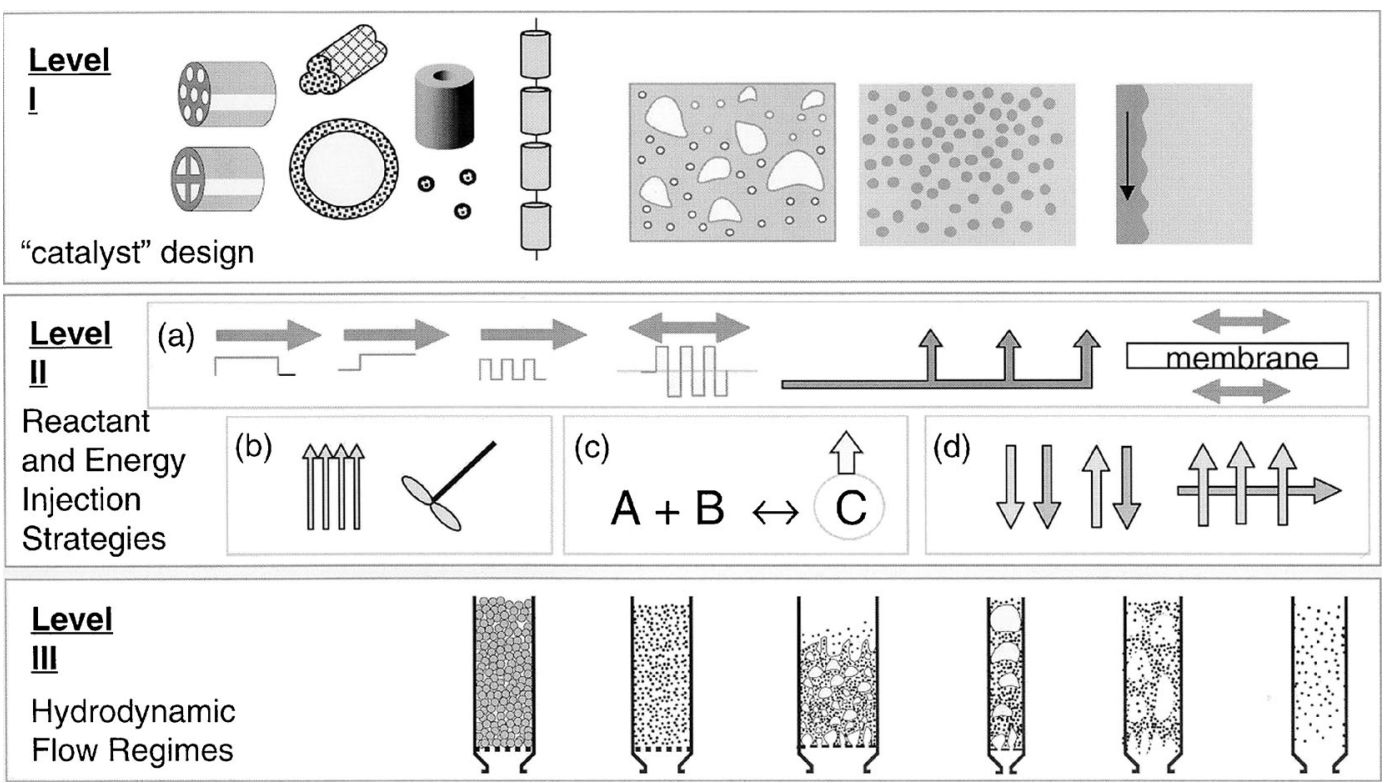

Figure 4. Strategies for multiphase reactors. ${ }^{10}$ 


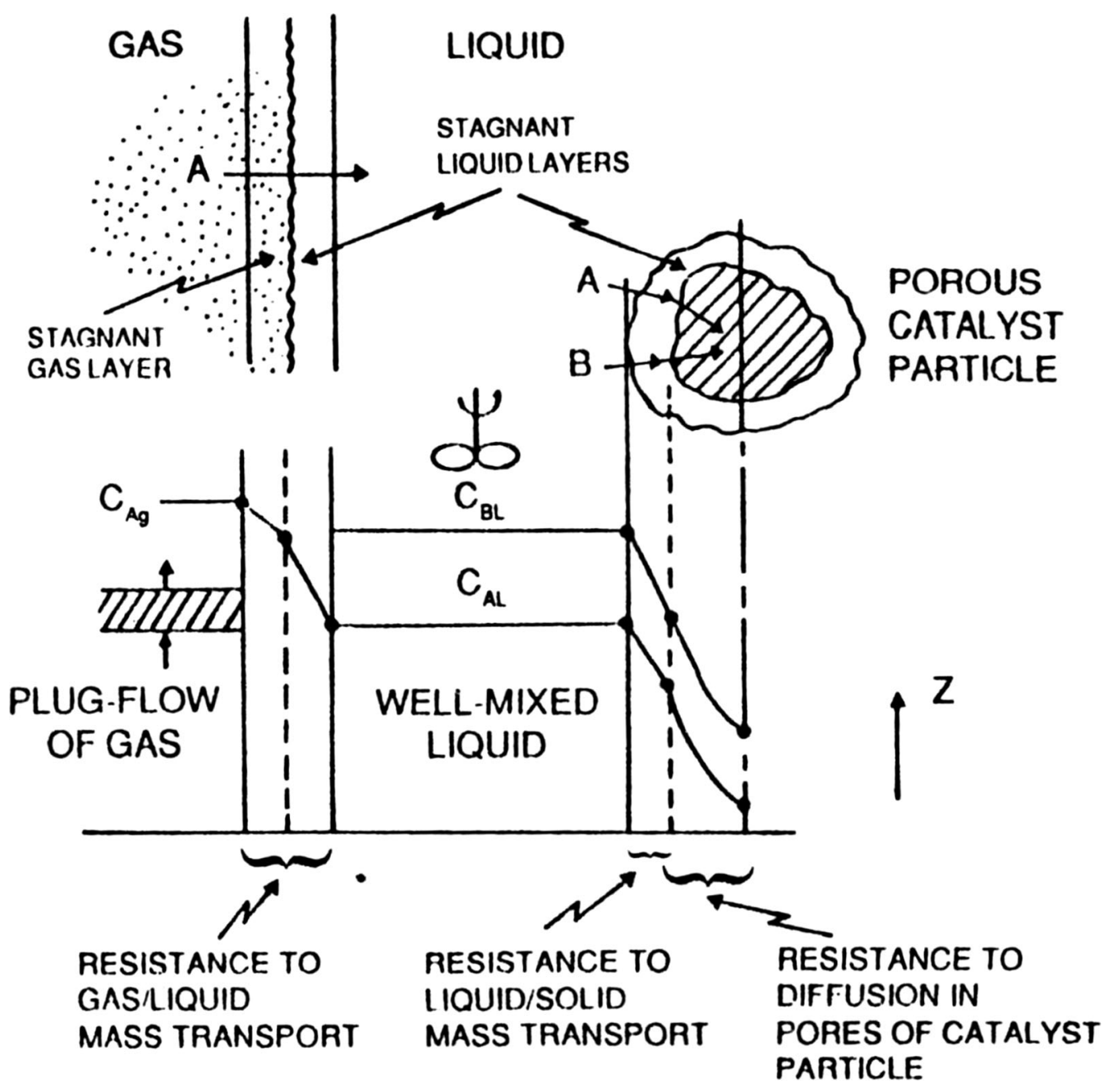

Figure 5. Gas-liquid and liquid-solid transport processes in catalytic reactors. ${ }^{5}$

are porous and therefore species and heat transport within the pores of catalyst particle control concentration and temperature profiles within the catalyst particle (and therefore conversion and selectivity). A schematic of pore diffusion and a way to quantify its influence via Thiele modulus and effectiveness factor is shown in figure 6. There are several ways by which effective Thiele modulus is defined to account for different shapes of catalyst pellets and different reaction orders. Interested reader may refer to Levenspiel (1999a). ${ }^{4}$

- Compensating inhibition/deactivation of catalyst: Various possible reasons for catalyst deactivation are mentioned earlier. Catalyst activity may get reduced due to deposition of inhibitors on active sites. Inhibitors may get consumed in reactions unlike catalysts. The most commonly used strategies with which one may compensate for reduced activity of catalyst are: by reducing flow rate or increasing temperature to maintain conversion at design level.
- Manipulate selectivity of desired product: Several strategies for enhancing selectivity of desired products have been proposed using classical CRE approach. These include manipulation of operating temperature or temperature profile across the reactor according to difference in activation energies of competing reactions (use high temperature if activation energy of reaction producing desired product is higher than reactions producing by-products). Several possible ways of enhancing selectivity by manipulating pore sizes of catalyst are discussed by Worstell (2001) and may be followed. ${ }^{11}$

For translating this understanding into practice, more often than not, key obstacles are lack of knowledge on how flow-patterns and contacting influence process performance and how these change with reactor scale (figure 7). ${ }^{12}$ It is impossible to provide detailed quantitative treatment to issues discussed earlier in this article. The cited references may be consulted for details on quantitative treatment. Some of the points 

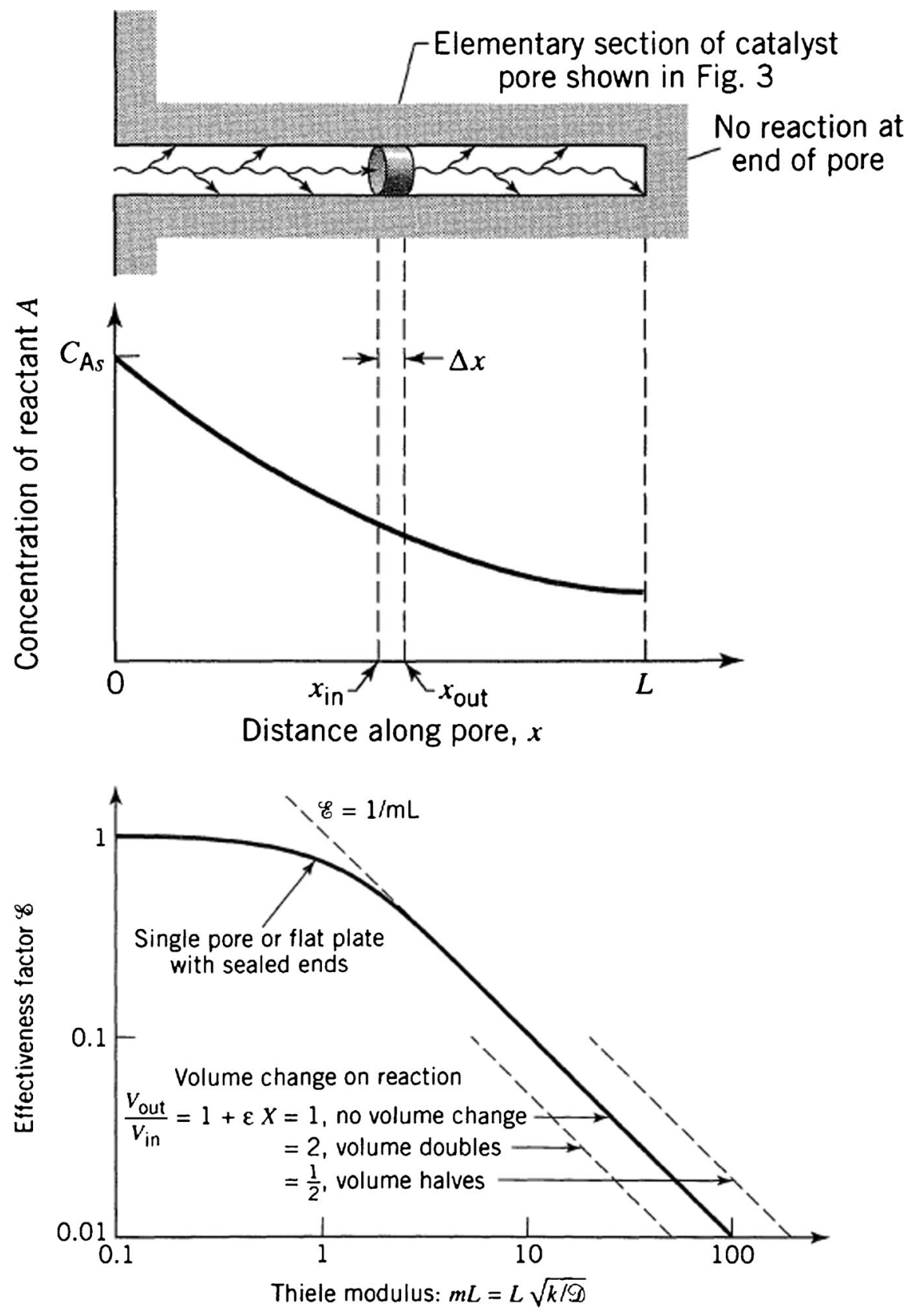

Figure 6. Pore diffusion and effectiveness factor. ${ }^{4,11}$

mentioned in this section are discussed with the help of illustrative examples and case studies in the following section.

\section{Illustrative examples and case studies}

\subsection{Pellet shape}

In practice, several shapes of catalyst pellets are used besides spheres and cylinders. For example, in cases where effectiveness factors are low, catalyst particles are 'designed' to increase external surface area. Various types of catalysts have been designed with internal holes which increase surface area and bed porosity for improving performance (better catalyst activity and lower pressure drop). Some of these particle shapes are shown in figure 8 a. Significant modelling and experimental studies has been conducted to understand influence of pellet shape on performance. As an example, studies by Dixon and coworkers ${ }^{13,14}$ are cited here. Typical simulated results of flow around pellets obtained by these researchers are shown in figure $8 \mathrm{~b}$. It 


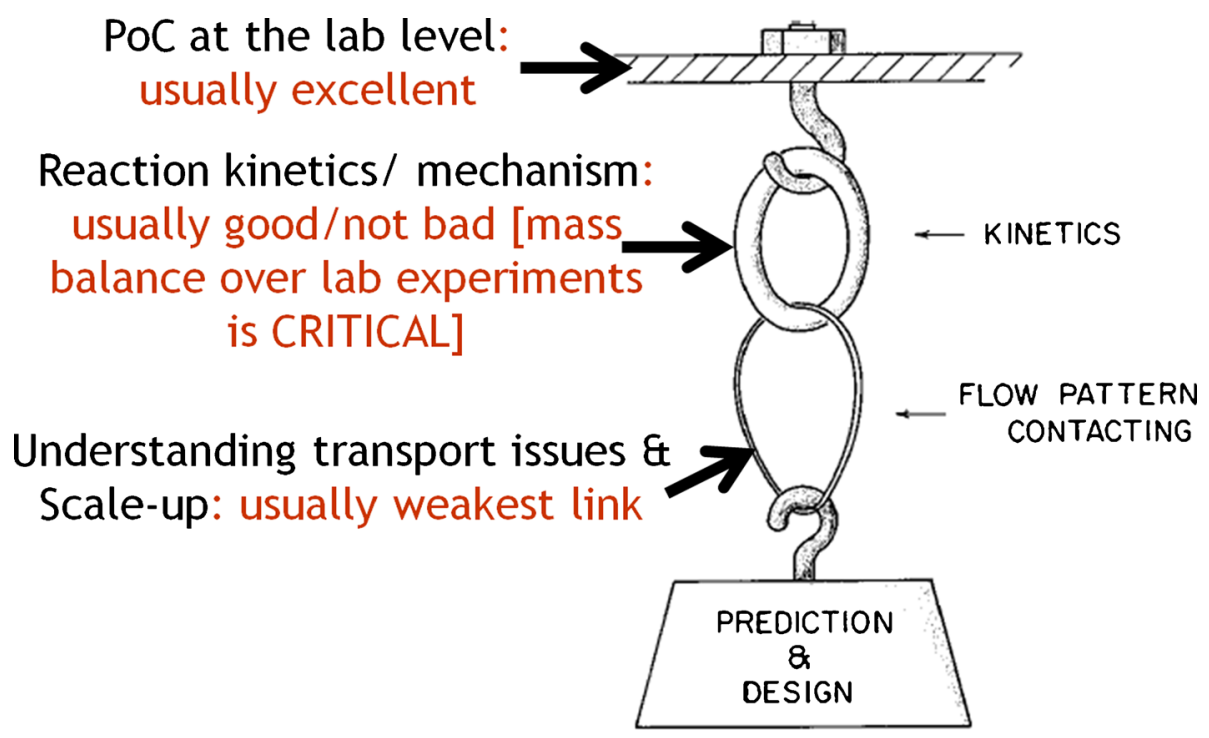

Figure 7. Practical reactor engineering (adapted from ${ }^{11}$ ).

was observed that for comparable pressure drop costs, multi-holed particles lead to a lower tube wall temperature. The influence of changing diameter of holes for the four-small-holes and one-big-hole particles on flow and heat transfer was found to be rather small. The methodology discussed by Ranade et al. (2011) may be used to guide design of catalyst particles for realizing better heat and mass transfer characteristics without incurring penalty of higher pressure drops. ${ }^{15}$

\subsection{Fixed bed reactors}

Once the pellet shape is finalized, it is important to design a bed of these pellets to ensure that the
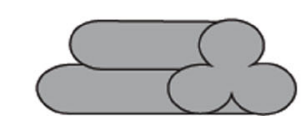

(a) Trilobe

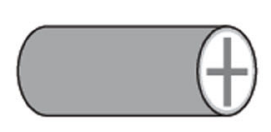

Wagon wheel

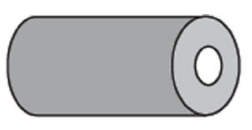

Hollow cylinder
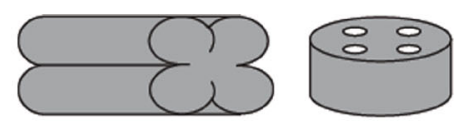

Quadrilobe

Four-hole cylinder
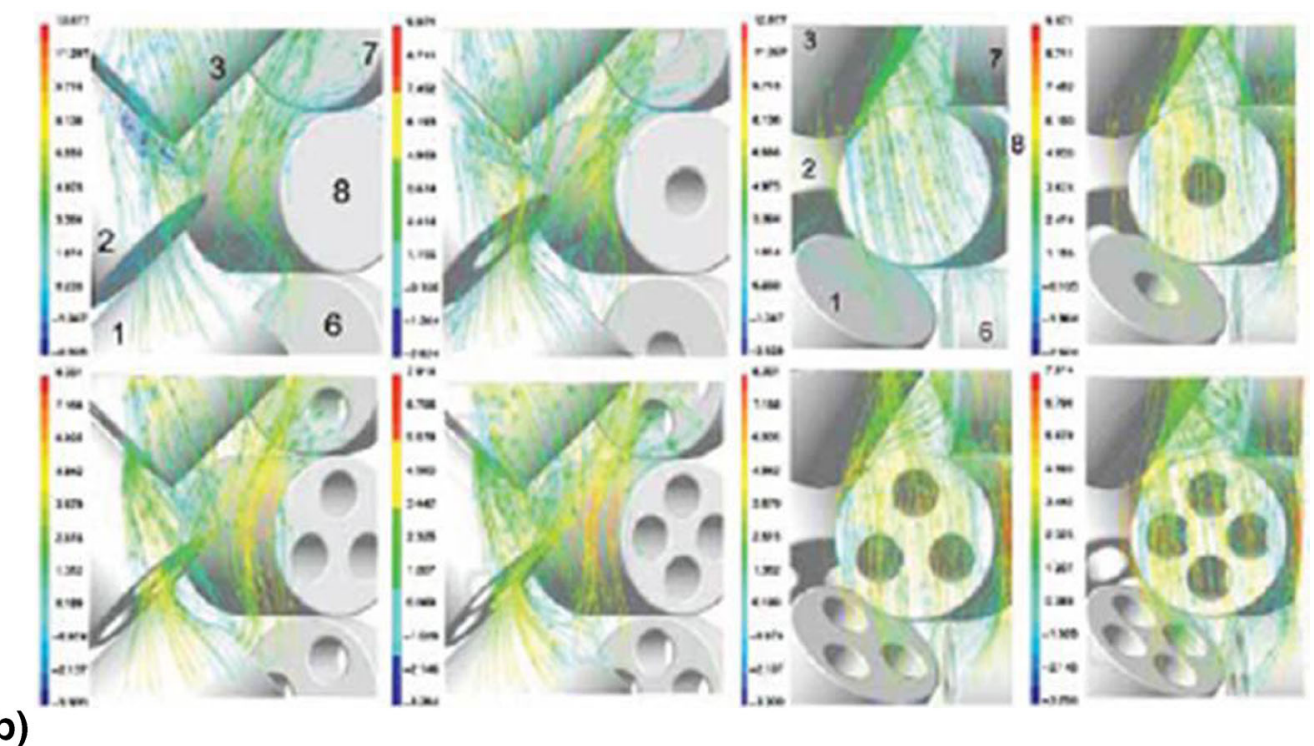

(b)

Figure 8. Influence of pellet shape on transport and reactor performance. (a) Different pellet shapes. ${ }^{15}$ (b) Flow around pellets. ${ }^{14}$ 
overall reactor performance is as desired. A typical example of vapour phase oxidation is considered here. In such vapour phase oxidation, mixing of hydrocarbon and oxygen plays a crucial role. This mixing has to be rapid to avoid a large region containing flammable mixture. Oxidation reactions are quite exothermic and may often lead to formation of local hot spots. Such hot spots may cause sintering and therefore deactivation of catalyst. Often, an inhibitor is added in the feed stream to reduce activity of catalyst to avoid formation of hot spots and to increase active life of catalyst. Many such vapour phase oxidation reactions are carried out in a shell and tube type of reactor to facilitate recovery of energy in the form of steam. Tube diameter is often critical to avoid hot spots. Classical reaction engineering models coupled with optimization are used for identifying optimal tube diameter. The design has to ensure that fed reactants and inhibitor, if any are distributed equally in all tubes. Note that many practical fixed bed reactors contain many bends in their feed network (see figure 9 for a typical feed network). The bends in feed network create many secondary flows and may cause severe mal-distribution within the reactor. The stateof-the-art computational models can help design engineers to quantify influence of bends in the feed network

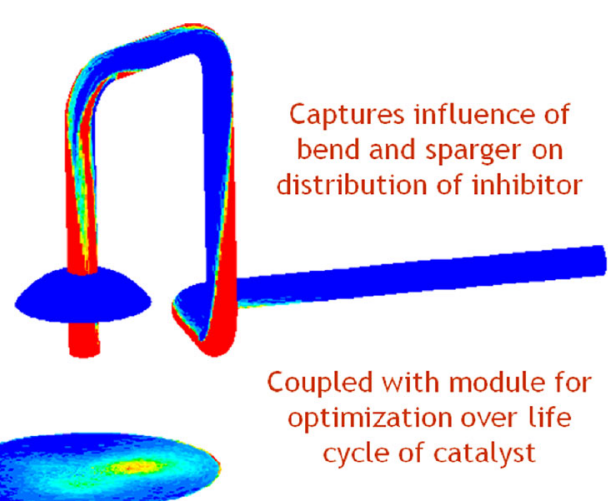

(a)

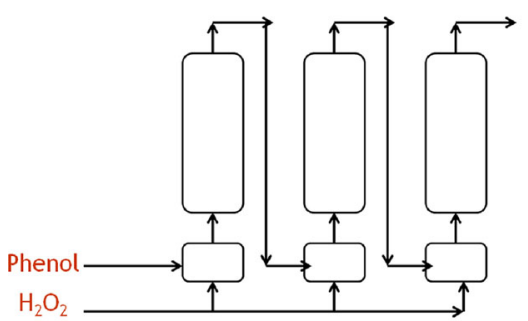

Select appropriate number of reactors and manipulate phenol to $\mathrm{H}_{2} \mathrm{O}_{2}$ ratio

(b)

Figure 9. Fixed bed reactors. (a) Maldistribution of inhibitor due to bends in feed network. (b) Multiple reactors and multiple dosing strategy for manipulating concentration profiles. and evolve ways to mitigate adverse influence. Contours shown in figure 9a show simulated distribution of inhibitor across the cross-section of fixed bed reactor. Computational models were then used to design guide vanes to be installed in the feed network and a distributor located in the dome above the catalyst bed to ensure that inhibitor is distributed evenly in the reactor.

Another example of a fixed bed reactor considered here is process developed at CSIR-NCL of hydroquinone using TS- 1 catalyst. The process involves the following reaction.

$$
\mathrm{C}_{6} \mathrm{H}_{5} \mathrm{OH}+\mathrm{H}_{2} \mathrm{O}_{2} \rightarrow \mathrm{C}_{6} \mathrm{H}_{4}(\mathrm{OH})_{2}+\mathrm{H}_{2} \mathrm{O}
$$

A typical fixed bed reactor produces two hydroxyl benzenes: hydroquinone and catechol. Hydroquinone is a desired product since it fetches much higher price than catechol. Selectivity of hydroquinone can be enhanced by appropriately manipulating local concentrations of phenol and hydrogen peroxide. The key degree of freedom available to a reactor engineer to manipulate local concentration profiles is multiple dosing of hydrogen peroxide so that local stoichiometric ratio is within the desirable range. A typical solution used in such cases is schematically shown in figure $9 \mathrm{~b}$.

\subsection{Slurry reactors}

For an active catalyst where required catalyst loading is quite small and usually liquid phase is required to provide adequate heat management, slurry reactors are used. Various slurry reactors such as stirred reactors (with or without gas inducing impellers), ejector loop reactor, and oscillating baffle reactor are used in practice. These reactors are often equipped with external heat exchanger to establish adequate management of heat liberated during reaction.

A typical example of slurry reactor application is hydrogenation. The example considered here is the production of para amino phenol from nitro benzene.

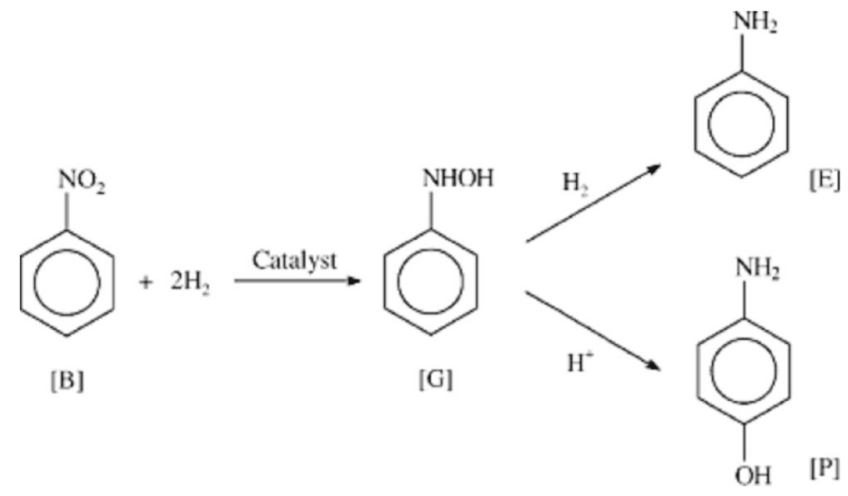


Obviously, para amino phenol $[\mathrm{P}]$ is a desired product since it fetches much higher price than aniline [E]. Selectivity of $[\mathrm{P}]$ can be enhanced by manipulating stoichiometric ratios of reactants, catalyst concentration, operating pressure (partial pressure of hydrogen), mass transfer/temperature profile and using multiple reactors. Larger catalyst concentration often favours aniline formation. Higher mass transfer offered by a jet loop reactor or a oscillatory baffle reactor may not always lead to higher selectivity since selectivity depends on rate controlling step. In such series-parallel reactions, it is often preferable to use multiple reactors operating at different conditions to favour the desired path of reactions.

Another example of slurry reactor considered here is of reductive alkylation with bubble column slurry reactor. ${ }^{16}$ The reaction scheme is as follows.

Careful laboratory experiments can be used to estimate kinetics of various reactions occurring in this complex reaction network. Knowledge of kinetics allows a reactor engineer to manipulate temperature and concentration profiles. One of the ways of manipulating concentration profiles is by manipulating backmixing in the reactor. For bubble column slurry reactor, the sparged gas often leads to churn turbulent flow leading to significant backmixing. Appropriate combination of reaction engineering models coupled with state-of-the-art computational fluid dynamics (CFD) models allows a reactor engineer to design appropriate reactor hardware to minimize backmixing. In this example, Chaudhari et al. (2007) have used CFD models to design radial baffles (see figure 10), which reduce backmixing and enhanced yield of desired product (by increasing conversion without affecting selectivity). ${ }^{16}$

Similar examples of applications of reactor engineering principles described here are observed commonly in industrial practice. A reactor engineer therefore has to draw useful lessons from these and use the basics of reaction engineering and flow modelling creatively to evolve best possible reactor hardware and operating protocols for reactions of interest. Recent efforts and advances in developing MAGIC (modular, agile, intensified and continuous) processes (see Ranade, ${ }^{18}$ and www.indusmagic.org for more information) need to be leveraged to develop better catalytic reactors and catalytic processes in practice.

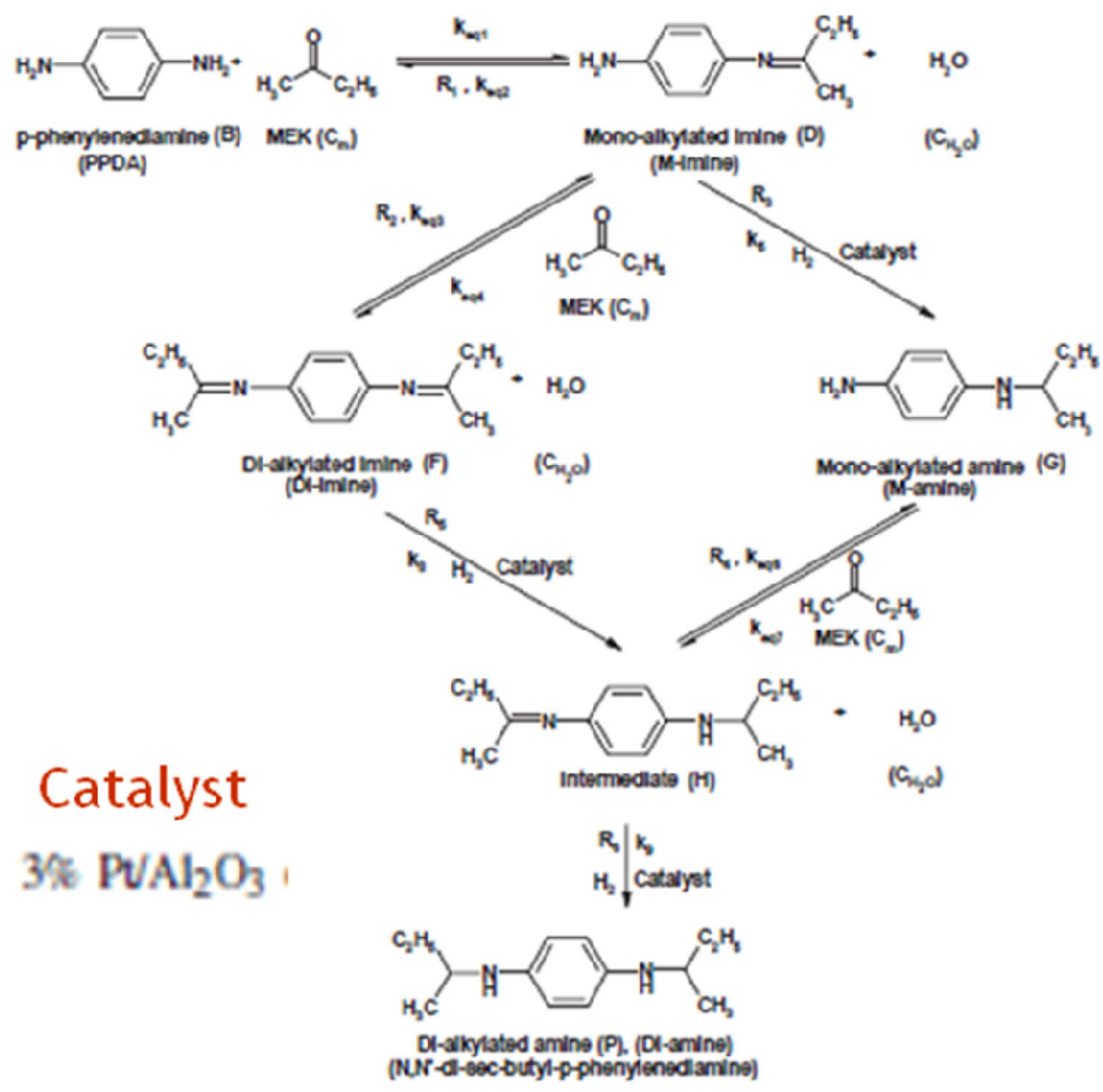




\section{Radial baffles to reduce backmixing}
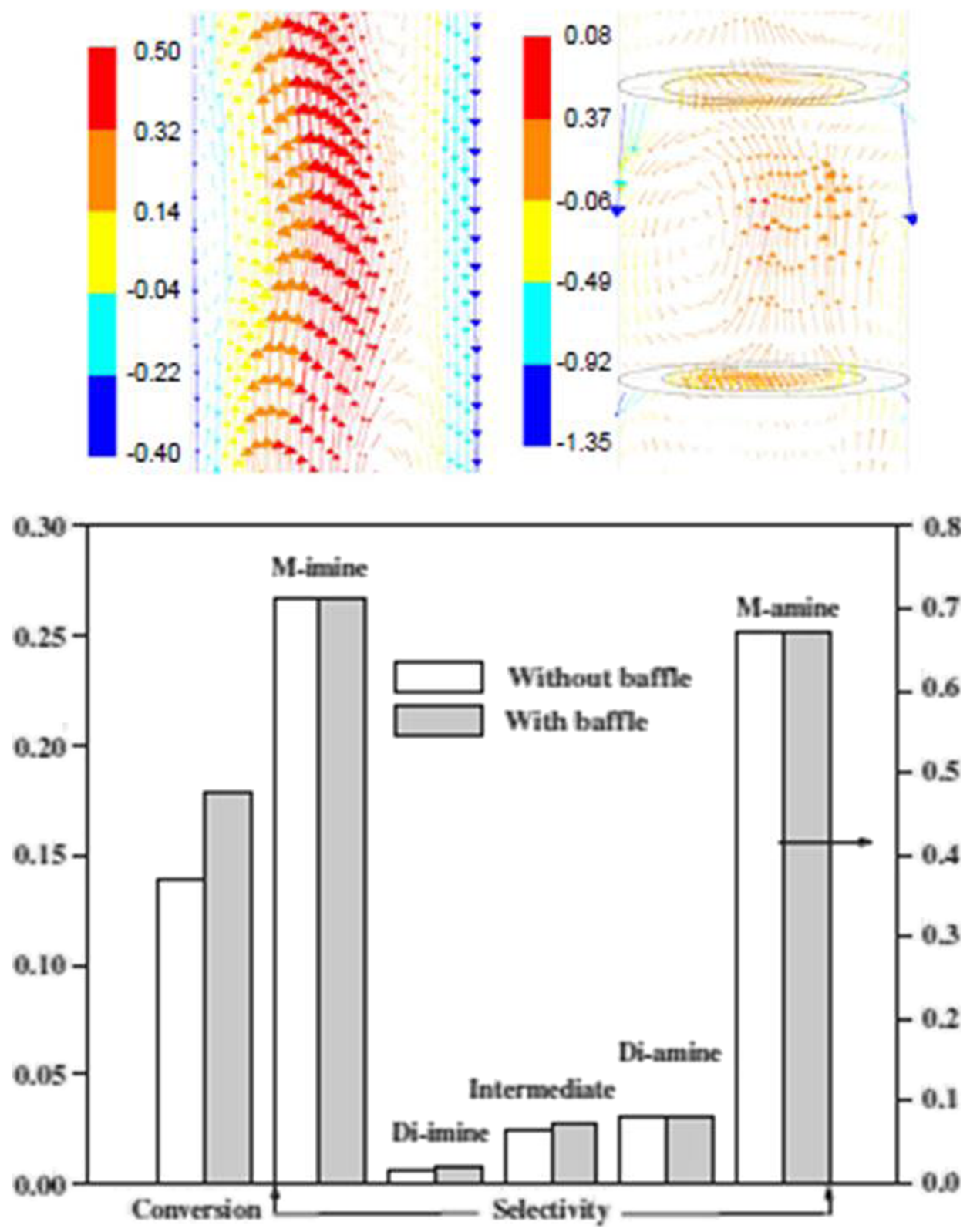

Figure 10. Bubble column slurry reactor - example of reductive alkylation. ${ }^{16}$

\section{Summary and conclusion}

Catalysts and catalytic reactions make substantial impact on process viability and economics. This article outlines recent (and not so recent) developments in engineering reactors. Catalyst design, appropriate choice of reactor, number of reactors, better injection and dispersion strategies and recent advances in process intensification should be harnessed to realize performance enhancement in practice. This article and cited examples may stimulate further interest and research in this important area.

\section{Acknowledgements}

Author thanks the Council of Scientific and Industrial Research (CSIR), New Delhi for financial support through Indus MAGIC (Innovate, develop and up-scale modular, agile, intensified and continuous processes) project. 


\section{References}

1. Aris R 1965 Introduction to the analysis of chemical reactors (Englewood Cliff, NJ: Prentice Hall Inc)

2. Westerterp K R, Van Swaaij W P M and Beenackers A A C M 1984 Chemical reactor design \& operation, 2nd edn (New York: John Wiley \& Sons)

3. Naumann E B 1987 Chemical reactor design (New York: John Wiley and Sons)

4. Levenspiel O 1999a Chemical reaction engineering, 3rd edn, Wiley

5. Ramchandran P A and Choudhari R V 1983 Three phase catalytic reactors (New York: Gordon and Breach)

6. Doraiswamy L K and Sharma M M 1984 Heterogeneous reactions - analysis examples and reactor design. Vol 2 (New York: John Wiley \& Sons)

7. Kunni D and Levenspiel O 1991 Fluidization engineering (New York: John Wiley \& Sons)
8. Shah Y T 1991 Adv. Chem. Eng. 17, 1

9. Dudukovic M P, Larachi F and Mills P L 1999 Chem. Eng. Sci. 54, 1975

10. Krishna R and Sie S T 1994 Chem. Eng. Sci. 49, 4029

11. Levenspiel O 1999b Ind. Eng. Chem. Res. 38, 4140

12. Worstell J H 2001 Chem. Eng. Progr. March, 68

13. Nijemeisland M and Dixon A G 2004 AIChE J. 50(5), 906

14. Dixon A G, Taskin M E, Nijemeisland M and Stit E H 2008 Chem. Eng. Sci. 63(8), 2219

15. Ranade V V, Chaudhari R V and Gunjal P R 2011 Trickle bed reactors (Elsevier: Academic Press)

16. Chaudhari A S, Rampure M R, Ranade V V, Jaganathan R and Chaudhari R V 2007 Chem. Eng. Sci. 62, 7290

17. Ranade V V 2002 Computational flow modelling for chemical reactor engineering (London: Academic Press)

18. Ranade V V 2012 June, Chemical News, 52 